\title{
Predicción de la resistencia térmica de Escherichia coli 0157: H7 en carne molida de res en función de la temperatura y las concentraciones de carvacrol y grasa
}

\author{
Prediction of the Escherichia coli 0157: $\mathrm{H} 7$ thermal resistance in ground beef as a function \\ of temperature, carvacrol and fat concentrations
}

\begin{abstract}
Jorge Isaac López-Pino, Martin Valenzuela-Melendres*, Juan Pedro Camou, Humberto González-Ríos, Fernando Ayala-Zavala, Aida Peña-Ramos

Coordinación de Tecnología de Alimentos de Origen Animal, Centro de Investigación en Alimentación y Desarrollo, A.C. Carretera Gustavo E. Astiazarán Rosas No. 46. Hermosillo, Sonora, 83304, México.

† A la memoria del Dr. Juan Pedro Camou
\end{abstract}

\section{RESUMEN}

Escherichia coli 0157: H7 es uno de los patógenos más importantes que contaminan la carne y comprometen su seguridad y calidad. Los tratamientos térmicos son la opción principal para garantizar la inocuidad; aunque el uso de barreras adicionales como la adición de agentes antimicrobianos, aumentan los márgenes de seguridad contra los patógenos. En este trabajo se evaluó el efecto y las interacciones de temperatura $\left(55-65^{\circ} \mathrm{C}\right)$, carvacrol $(0-2 \%)$ y grasa $(5-$ 20\%) sobre la resistencia térmica (valor D) de Escherichia coli 0157: H7 en carne molida de res utilizando la metodología de superficie de respuesta. La temperatura fue el principal factor de inactivación y la grasa mostró un efecto protector sobre el patógeno. Hubo un efecto de interacción entre la grasa y el carvacrol, a altas concentraciones de grasa el carvacrol disminuyó la resistencia del patógeno. La combinación óptima de grasa, carvacrol y temperatura que minimizan los valores $\mathrm{D}$ fue $63{ }^{\circ} \mathrm{C}, 11 \%$ de grasa y $1.6 \%$ de carvacrol. El modelo obtenido permite predecir la resistencia térmica de E. coli 0157: $\mathrm{H} 7$ en cualquier combinación de temperatura, carvacrol y grasa, dentro de los límites estudiados.

Palabras clave: Resistencia térmica, antimicrobianos naturales, inocuidad de la carne, metodología de superficie de respuesta.

\section{ABSTRACT}

Escherichia coli 0157: $\mathrm{H} 7$ is one of the most important pathogens in ground beef, and the risk of contamination compromises its safety and quality. Thermal treatments are the main option to ensure food safety; although, the use of additional barriers such as the addition of antimicrobial agents increases safety margins against pathogens growth. In this work the effects and interactions of temperature (55$\left.65^{\circ} \mathrm{C}\right)$, carvacrol (0-2\%) and fat (5-20\%) on thermal resistance (D-value) of E. coli 0157: $\mathrm{H} 7$ in ground beef were evaluated using response surface methodology. Temperature was the main factor of inactivation, while fat exhibited a protective effect over the pathogen survival. There was an interaction effect between fat and carvacrol, at high concentrations of fat the carvacrol favored the inactivation of the pathogen. The optimal combination of fat, carvacrol and temperature that ensure the highest inactivation of the pathogen was 63 ${ }^{\circ} \mathrm{C}, 11 \%$ fat and $1.6 \%$ carvacrol. The obtained model allow to predict the thermal resistance of E. coli O157: $\mathrm{H} 7$ in any combination of temperature, carvacrol and fat, within the limits studied.

Key words: Thermal resistance, natural antimicrobials, meat safety, response surface methodology.

\section{INTRODUCCIÓN}

La carne es una fuente excelente de proteínas de alta calidad biológica, vitaminas y minerales de gran importancia para mantener una buena salud física en todas las etapas de la vida (Cashman y Hayes, 2017). La producción de carne de res en México es cercana a los 2 millones de toneladas anuales con un consumo per cápita de 15 kg (Ríos-Flores y CastilloArce, 2015). La carne fresca de res se vende al consumidor en forma de bistec, filete y carne molida, principalmente. Esta última es la base para la preparación de diferentes platillos como hamburguesas, picadillo y albóndigas, tanto en el hogar como en establecimientos comerciales para su venta al público.

Escherichia coli 0157: H7 es uno de los patógenos más importantes que contaminan la carne (Kim et al., 2020). Esta bacteria se encuentra con frecuencia en el intestino del ganado vacuno, llega a la superficie de la canal durante el desollado y evisceración a través del equipo y las manos de las personas que manipulan las canales, y es incorporado en la matriz cárnica durante el proceso de molienda, por eso la mayoría de los casos de infección se han relacionado con el consumo de carne molida cocinada inadecuadamente (CDC, 2020). El Centro para el Control y Prevención de Enfermedades de los Estados Unidos reporta anualmente más de 95,000 casos de infecciones por E. coli O157: H7, y aunque la carne es la principal fuente de infección, alimentos como ensaladas, lechuga romana, leches y jugos sin pasteurizar, son también fuentes importantes (CDC, 2020). 
Para asegurar la inocuidad de la carne, el Departamento de Agricultura de los Estados Unidos recomienda cocinar la carne molida hasta alcanzar una temperatura de $71.1^{\circ} \mathrm{C}$ (USDA-FSIS, 2020), medida ésta por medio de un termómetro. Sin embargo, su uso no es tan común, siendo el color visual de la carne el método más extendido para juzgar su cocimiento, a pesar de que éste no garantiza la inocuidad del producto (Lin, 2018). Se ha reportado que, a una temperatura interna de cocimiento de $57^{\circ} \mathrm{C}$, el color visual de la carne es similar al de una completamente cocida, pero es insuficiente para eliminar bacterias patógenas (Lyon et al., 2000).

El uso de barreras adicionales como la adición de antimicrobianos permiten dar una mayor confianza al tratamiento con calor y dar un margen de seguridad mayor contra los principales microorganismos contaminantes de la carne (Surendran Nair et al., 2016). El carvacrol es un fenol monoterpénico producido por varias plantas aromáticas, principalmente orégano. Se utiliza en concentraciones bajas como ingrediente aromatizante y condimento de los alimentos, y ejerce una importante actividad antimicrobiana contra los patógenos transmitidos por los alimentos (Scandorieiro et al., 2016). El carvacrol provoca la formación de poros en la bicapa lipídica a través de los cuales se crea un flujo de protones hacia el exterior celular. La formación de los poros trae como consecuencias la desestabilización de la membrana debido a la inserción del carvacrol y la ruptura del equilibrio iónico causado por la salida de los protones. Para aumentar los márgenes de seguridad durante el tratamiento térmico, se ha utilizado el carvacrol en sinergia con el calor para la inactivación de Salmonella en carne de pollo (Juneja et al., 2012), así como también la inactivación de E. coli O157: $\mathrm{H} 7$ y Clostridium perfringes en carne molida de res (Juneja y Friedman, 2008; Juneja et al., 2006).

La carne molida de res usualmente contiene grasa en cantidades que oscilan entre 5 y $20 \%$, siendo ésta última la más económica y popular. Se ha demostrado que la grasa en la carne ejerce un efecto protector contra patógenos. La grasa reduce la transferencia de calor y aumenta los tiempos de exposición a una temperatura dada, disminuyendo así la efectividad del tratamiento térmico (Juneja et al., 2001).

En la mayoría de las investigaciones reportadas se estudian en forma separada los diversos factores involucrados en la inactivación de microorganismos patógenos, pasando por alto posibles interacciones entre ellos. Estas interacciones ayudan a comprender las relaciones entre los componentes de la matriz cárnica que se dan de manera natural. Para la descripción del proceso de inactivación térmica no es suficiente conocer la resistencia del patógeno a la temperatura o a los antimicrobianos, sino que también es necesario saber cómo es la interacción del patógenos con todos los componentes de la matriz cárnica y como estos pueden influir en la supervivencia de los microorganismos funcionando como barreras protectoras como en el caso de la grasa. La incorporación de un mayor número de componentes de la matriz permite recrear mejor como se llevan a cabo los procesos de contaminación por parte de los patógenos y el papel de cada componente en su protección o inhibición. Estudiar los efectos combinados del calor, antimicrobianos naturales y contenido de grasa sobre la resistencia de patógenos, puede volverse una tarea complicada si no se utiliza la estrategia metodológica adecuada. Los modelos de predicción son herramientas útiles que facilitan el estudio de múltiples factores que afectan la resistencia térmica de los patógenos. La metodología de superficie de respuesta es una herramienta matemática y estadística utilizada para evaluar varios factores al mismo tiempo y estimar sus efectos lineales, cuadráticos y de interacción (Mongomery, 2013). El objetivo del presente trabajo fue estudiar la resistencia térmica de E. coli O157: H7 en carne molida de res en función de la temperatura y las concentraciones de carvacrol y grasa utilizando la metodología de superficie de respuesta.

\section{MATERIALES Y MÉTODOS Diseño experimental}

Se utilizó un diseño central compuesto (DCC) para determinar los efectos e interacciones de la temperatura ( $T$, en los niveles de $55,57,60,63$ y $65^{\circ} \mathrm{C}$ ), carvacrol ( $\mathrm{C}$, en los niveles de $0,0.4,1.0,1.6$ y $2.0 \%$ ) y grasa (G, en los niveles de 5,8 , $12.5,17$ y $20 \%$ ) sobre la resistencia térmica (valores D, tiempo en min para reducir el $90 \%$ de la población microbiana) de E. coli 0157: H7 inoculada en carne molida de res. Las corridas experimentales del DCC se formaron por ocho puntos factoriales, ocho puntos axiales a una distancia de \pm 1.68 del centro y seis réplicas del punto central para un total de 20 tratamientos. Fueron incluidos como control cinco tratamientos sin la adición de grasa y carvacrol (Tabla 1). Se realizaron dos repeticiones del experimento completo y cada combinación de tratamientos se realizó por duplicado en cada repetición.

\section{Cepas bacterianas y preparación del inóculo}

Se empleó la cepa ATCC 43890 de E. coli O157: H7 perteneciente al Laboratorio de Tecnologías Emergentes del Centro de Investigación en Alimentación y Desarrollo, A.C. de Hermosillo, Sonora. La cepa se conservó en viales a $-80^{\circ} \mathrm{C}$ en caldo cerebro-corazón (Labline-Thermo Scientific, Melrose Park, Illinois) con un 15\% (vol/vol) de glicerol.

Para la preparación del inóculo se siguió la metodología de Juneja y Novak (2003). Se descongeló el vial que contenía la cepa y se transfirieron $0.1 \mathrm{~mL}$ del cultivo descongelado a $10 \mathrm{~mL}$ de caldo cerebro-corazón, seguido de $24 \mathrm{~h}$ de incubación a $37{ }^{\circ} \mathrm{C}$ (Labline-Thermo Scientific, Melrose Park, Illinois). Este procedimiento se realizó por duplicado y posteriormente se tomó $0.1 \mathrm{~mL}$ del cultivo para ser inoculado en tubos con $50 \mathrm{~mL}$ de caldo cerebro-corazón e incubado a $37{ }^{\circ} \mathrm{C}$ por $24 \mathrm{~h}$.

Para la preparación de la suspensión de bacterias a emplear en el ensayo se tomó $0.1 \mathrm{~mL}$ del cultivo y se transfirió a un matraz que contenía $50 \mathrm{~mL}$ de caldo cerebrocorazón y se incubó por $24 \mathrm{~h}$ a $37^{\circ} \mathrm{C}$. Pasadas $24 \mathrm{~h}$ los cultivos fueron centrifugados (Eppendorf, modelo 5810R, Hamburgo, Alemania) a $5,000 \times \mathrm{g}$ durante $15 \mathrm{~min}$ a $4{ }^{\circ} \mathrm{C}$, se decantó el sobrenadante y se disolvió el precipitado en $9.9 \mathrm{~mL}$ de agua 
Tabla 1. Valores codificados y experimentales de los tratamientos resultantes del diseño central compuesto

Table 1. Coded and experimental values of treatments from the central composite design

\begin{tabular}{|c|c|c|c|c|c|c|}
\hline \multirow[b]{2}{*}{ Corridas } & \multicolumn{3}{|c|}{ Valores Codificados } & \multicolumn{3}{|c|}{ Valores Experimentales } \\
\hline & $x_{1}$ & $x_{2}$ & $x_{3}$ & $\begin{array}{c}\text { Temperatura } \\
\left({ }^{\circ} \mathrm{C}\right)\end{array}$ & $\begin{array}{c}\text { Grasa } \\
(\%)\end{array}$ & $\begin{array}{c}\text { Carvacrol } \\
(\%)\end{array}$ \\
\hline 1 & -1 & -1 & -1 & 57 & 8 & 0.4 \\
\hline 2 & 1 & -1 & -1 & 57 & 8 & 1.6 \\
\hline 3 & -1 & 1 & -1 & 57 & 17 & 0.4 \\
\hline 4 & -1 & -1 & 1 & 57 & 17 & 1.6 \\
\hline 5 & 1 & 1 & -1 & 63 & 8 & 0.4 \\
\hline 6 & 1 & -1 & 1 & 63 & 8 & 1.6 \\
\hline 7 & -1 & 1 & 1 & 63 & 17 & 0.4 \\
\hline 8 & 1 & 1 & 1 & 63 & 17 & 1.6 \\
\hline 9 & $-a$ & 0 & 0 & 55 & 12.5 & 1.0 \\
\hline 10 & $a$ & 0 & 0 & 65 & 12.5 & 1.0 \\
\hline 11 & 0 & $-a$ & 0 & 60 & 5 & 1.0 \\
\hline 12 & 0 & $a$ & 0 & 60 & 20 & 1.0 \\
\hline 13 & 0 & 0 & $-a$ & 60 & 12.5 & 0 \\
\hline 14 & 0 & 0 & $a$ & 60 & 12.5 & 2.0 \\
\hline 15 & 0 & 0 & 0 & 60 & 12.5 & 1.0 \\
\hline 16 & 0 & 0 & 0 & 60 & 12.5 & 1.0 \\
\hline 17 & 0 & 0 & 0 & 60 & 12.5 & 1.0 \\
\hline 18 & 0 & 0 & 0 & 60 & 12.5 & 1.0 \\
\hline 19 & 0 & 0 & 0 & 60 & 12.5 & 1.0 \\
\hline 20 & 0 & 0 & 0 & 60 & 12.5 & 1.0 \\
\hline 21 & $-a$ & $-a$ & $-a$ & 55 & 5 & 0 \\
\hline 22 & -1 & $-a$ & $-a$ & 57 & 5 & 0 \\
\hline 23 & 0 & $-a$ & $-a$ & 60 & 5 & 0 \\
\hline 24 & 1 & $-a$ & $-a$ & 63 & 5 & 0 \\
\hline 25 & $a$ & $-a$ & $-a$ & 65 & 5 & 0 \\
\hline
\end{tabular}

Las corridas 1-8, son puntos factoriales, las 9-14 son puntos axiales, las 1520 son puntos centrales, y las 21-25 son puntos control del DCC.

de peptona 1\% de (BD Diagnostics Systems, Sparks, MD), para una nueva centrifugación a las mismas condiciones. El precipitado fue finalmente disuelto en $2 \mathrm{~mL}$ de agua de peptona al $1 \%$ obteniendo un inóculo de aproximadamente 8 Log UFC/mL que se empleó para inocular la muestra.

\section{Materiales de ensayo y preparación de los tratamientos}

Los tratamientos incluyeron carne de res (músculo Semimembranosus), grasa dorsal y carvacrol de origen natural grado alimenticio con $98 \%$ de pureza (Sigma-Aldich ${ }^{\oplus}$, Darmstadt, Germany). Tanto la carne como la grasa fueron obtenidos del comercio local y utilizadas el mismo día de la adquisición. Se cortaron en piezas no mayor a $5 \mathrm{~cm} 3$ y se molieron en lotes por separado usando un molino Hobart (modelo 4152, Troy, Ohio, USA) a través de una malla con orificios de $4.8 \mathrm{~mm}$. La carne, grasa y carvacrol fueron mezcladas manualmente durante $1 \mathrm{~min}$, seguido de un mezclado mecánico (Stomacher 80, Lab System, Steward) durante 1 min adicional. La combinación de tratamientos se realizó de acuerdo a lo mostrado en la Tabla 1. Se tomaron porciones de $5.0 \pm 0.1 \mathrm{~g}$ de cada tratamiento y se colocaron en bolsas estériles para vacío de $9 \times 14 \mathrm{~cm}$ (Whirl Pak ${ }^{\oplus}$, USA). Las muestras se congelaron $\mathrm{a}-40^{\circ} \mathrm{C}$ hasta su uso posterior.

\section{Tratamiento térmico y enumeración de bacterias super- vivientes}

En los días de prueba, las muestras fueron descongeladas a temperatura ambiente e inoculadas con $0.1 \mathrm{~mL}$ de la solución bacteriana en agua de peptona $1 \%$ para obtener una concentración aproximada de 7 log UFC/g. Posteriormente se mezcló en el stomaker (Stomacher 80, Lab System, Steward) durante 1 min para lograr una distribución homogénea de la bacteria en el producto. Después de esto, cada bolsa se comprimió en una capa delgada ( 0.5 a $1 \mathrm{~mm}$ de espesor) para conseguir una distribución de calor uniforme en la muestra y excluir el aire presente dentro de la bolsa antes de sellarla térmicamente.

Las muestras preparadas para cada tratamiento se colocaron en una gradilla y se sumergieron en un baño de agua con recirculación (ThermoFisher Scientific Neslab Instruments, Inc., Newington, NH) con una temperatura fijada según el DCC de la Tabla 1. Los tiempos para alcanzar la temperatura de prueba en el centro del producto fueron menores a $5 \mathrm{~s}$, por lo que se incluyeron en el tiempo total de calentamiento para el cálculo de los valores $\mathrm{D}$ (tiempo para inactivar el $90 \%$ de las células bacterianas viables).

El intervalo de tiempo para el muestreo se estableció en función de la exposición y osciló entre $15 \mathrm{~min}$ a $55^{\circ} \mathrm{C}$ y $10 \mathrm{~s}$ a $65^{\circ} \mathrm{C}$. Después del tratamiento térmico, las muestras se sumergieron en un baño de hielo-agua durante $10 \mathrm{~min}$ para lograr su rápido enfriamiento. Cada bolsa se abrió asépticamente; se añadieron $5 \mathrm{~mL}$ de agua de peptona $1 \%$ y se homogeneizaron para recoger la suspensión de líquido resultante de la interacción con la carne. Después de esto, se realizaron diluciones seriadas transfiriendo $0.1 \mathrm{~mL}$ del filtrado a $9.9 \mathrm{~mL}$ de agua de peptona, y se inocularon sobre agar para conteo en placas (Difco, BD, Sparks, MD), las cuales se dejaron en reposo por $2 \mathrm{~h}$ para luego cubrirlas con una capa de agar MacConkey-sorbitol (Difco, BD, Sparks, MD). Las colonias se enumeraron después de $24 \mathrm{~h}$ de incubación a $37^{\circ} \mathrm{C}$ en una incubadora (Thermo Scientific, Heratherm IMH 180). Cada corrida experimental se realizó por duplicado y se utilizó la media de las UFC/g de dos placas de cada punto de muestreo para determinar la letalidad de la temperatura.

\section{Procesamiento de datos, y cálculo del valor $\mathrm{D}$ y cálculo del valor $z$}

Se utilizó el modelo Baranyi (Baranyi y Roberts, 1995) con el software de edición DMFit Excel (Institute of Food Research, Norwich, Reino Unido) para ajustar el logaritmo de las poblaciones microbianas y calcular los valores $D$ a partir de la pendiente de la parte lineal de la curva de inactivación. A continuación, los valores $D$ se transformaron a la forma de logaritmo natural (In) para estabilizar la varianza. Los valores D obtenidos fueron analizados con el paquete estadístico Design Expert para la obtención de los modelos de regresión en función de la temperatura de inactivación y contenidos de grasa y carvacrol.

Los valores $\mathrm{z}$ (incremento en la temperatura en ${ }^{\circ} \mathrm{C}$ necesaria para cambiar el valor $D$ en una unidad logarítmica) se 
estimaron a partir de la regresión lineal derivada del registro de los valores $D$ frente a sus temperaturas de calentamiento correspondientes, utilizando para ello el software Excel (Microsoft, Redmond, WA). Los valores z se calcularon como el valor absoluto del recíproco de la pendiente de la línea de regresión.

\section{Análisis estadístico}

Se utilizó la metodología de superficie de respuesta para establecer el modelo de regresión. Se realizó un análisis de varianza y se determinó el coeficiente de determinación $\left(R^{2}\right)$ y la falta de ajuste del modelo utilizando el paquete estadístico Design Expert 7.0.0 (Stat-Ease, Inc., Minneapolis, MN, USA). El modelo matemático correspondiente al DCC es:

$$
\mathrm{y}=\beta_{0}+\sum_{i=1}^{3} \beta_{i} X_{1}+\sum_{i=1}^{3} \beta_{i i} X_{i}^{2}+\sum_{i<j} \beta_{i j} X_{i} X_{j}+\varepsilon
$$

donde y es la variable respuesta (valor D), $\beta_{o^{\prime}} \beta_{i^{\prime}} \beta_{i i^{\prime}}$ y $\beta_{i j}$ son el intercepto y los coeficientes lineales, cuadráticos y de interacción, respectivamente; $X_{i-j}$ son las variables independientes (temperatura, carvacrol y grasa).

Para la optimización de la variable de respuesta (valor D), se utilizó la técnica de optimización numérica del software Design-Expert. En el presente trabajo se estableció la combinación óptima de temperatura de inactivación, contenido de grasa y contenido de carvacrol que minimizan el valor $\mathrm{D}$ durante el procesamiento de carne molida de res. Se preparó un lote de carne molida con los niveles óptimos de los ingredientes, se determinó el valor $D$ experimentalmente y se comparó estadísticamente $(p<0.05)$ con el valor $\mathrm{D}$ predicho por el modelo.

\section{RESULTADOS Y DISCUSIÓN}

\section{Efectos de la temperatura, grasa y carvacrol sobre el valor D}

Los efectos de la temperatura, grasa y carvacrol sobre la resistencia térmica de $E$. coli $0157: \mathrm{H} 7$ inoculada en carne molida de res se determinaron a partir del cálculo del valor D. La Figura 1 muestra un ejemplo de las curvas de muerte térmica para E. coli 0157: $\mathrm{H} 7$ que fue usada para calcular el valor D de acuerdo al modelo de Baranyi y Roberts (1995). El cálculo se realizó para cada una de las 2 réplicas de las 25 corridas experimentales y el promedio del valor D se utilizó para desarrollar el modelo de regresión aplicando la MSR. La Tabla 2 muestra los resultados experimentales para las 25 combinaciones de los tratamientos de acuerdo al DCC usado. El mayor valor estimado fue 14.45 min obtenido en la corrida 9 (axial) con la combinación de factores: $55^{\circ} \mathrm{C}, 12.5 \%$ grasa y $0 \%$ de carvacrol, y el más bajo fue de $0.17 \mathrm{~min}$ a partir de la combinación: $65^{\circ} \mathrm{C}, 5 \%$ de grasa y $0 \%$ de carvacrol en la corrida 25 (control).

Los efectos de la temperatura, la grasa y el carvacrol se observan en las corridas axiales mostradas en la Tabla 2. Por ejemplo, en las corridas 9 y 10 el valor $D$ disminuye desde $14.45 \mathrm{~min}$ hasta $0.18 \mathrm{~min}$ cuando la temperatura aumenta de

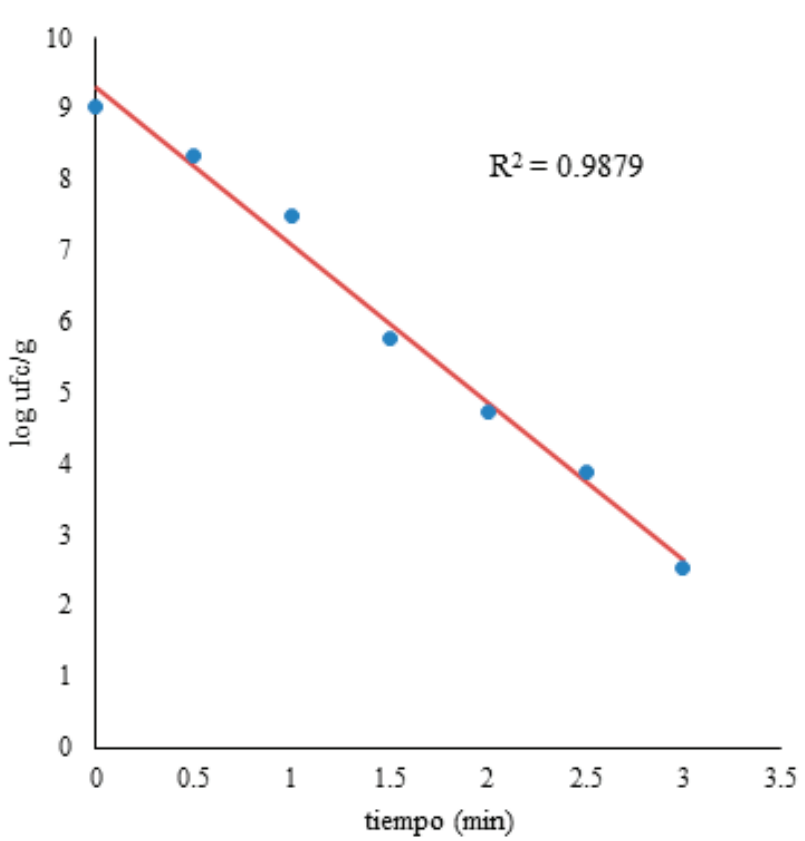

Figura 1. Ajuste de los datos experimentales por el modelo de Baranyi (línea continua) para la supervivencia de $E$. coli 0157: H7 (UFC/g) vs tiempo del tratamiento térmico correspondiente a la corrida 5 del DCC (temperatura $63^{\circ} \mathrm{C}$, grasa $8 \%$ y carvacrol $0.4 \%$ ).

Figure 1. Adjustment of experimental data by Baranyi model (continuous line) for the survival of $E$. coli $0157: \mathrm{H} 7(\mathrm{CFU} / \mathrm{g})$ vs heat treatment time corresponding to $C C D$ run 5 (temperature $63^{\circ} \mathrm{C}$, fat $8 \%$ and carvacrol $0.4 \%$ ).

55 a $65^{\circ} \mathrm{C}$ y la grasa y el carvacrol permanecen constantes. Cuando la grasa aumenta desde el 5 al $20 \%$ y la temperatura y el carvacrol permanecen constantes el valor $D$ aumenta de 0.88 hasta 2.14 min (corridas 11 y 12 , respectivamente), lo cual indica que existe un efecto protector de la grasa sobre la bacteria. Un incremento en la concentración de carvacrol de 0 a $2 \%$, disminuye la resistencia térmica de la bacteria como se muestra en las corridas 13 y 14 , manteniendo constantes la temperatura y la cantidad de grasa.

\section{Modelo de regresión para estimar el valor $D$}

La Tabla 3 muestra el coeficiente de regresión para cada uno de los términos del modelo, además de la significancia y el coeficiente de determinación $\mathrm{R}^{2} \mathrm{y}$ la falta de ajuste. De acuerdo al ANOVA el modelo es adecuado $(p<0.0001)$ para predecir la variable respuesta, el cálculo del coeficiente de determinación indica que el $98 \%$ de la variación total puede ser explicada con este modelo, además no hay falta de ajuste en él $(p=0.206)$. La Figura 2 muestra el grado de ajuste lineal entre los datos experimentales del valor $D$ y los valores predichos por el modelo estadístico. Los criterios anteriores muestran que el modelo es adecuado para describir los datos experimentales y puede ser empleado para predecir el valor $D$ en los límites establecidos para los factores temperatura $\left(55-65^{\circ} \mathrm{C}\right)$, grasa $(5-20 \%)$ y carvacrol (0-2\%). La ecuación del modelo de regresión se expone a continuación:

$\operatorname{Ln}($ Valor $D)=0.097-1.25 \boldsymbol{T}+0.18 \boldsymbol{G}-0.13 \boldsymbol{C}-0.1 \boldsymbol{G C}+0.16 \boldsymbol{T}^{2}+0.12 \boldsymbol{G}^{2}$ 
Tabla 2. Valores $\mathrm{D}(\mathrm{min})$ experimentales y predichos para $E$. coli 0157: H7 en carne molida de res con diferentes niveles de grasa y carvacrol, cocinadas a diferentes temperaturas Table 2. Experimental and predicted D-values for $E$. coli O157: H7 in ground beef with different levels of fat and carvacrol, cooked at different temperatures.

\begin{tabular}{|c|c|c|c|c|c|c|}
\hline \multirow{3}{*}{ Corrida $^{a}$} & \multirow{3}{*}{$\begin{array}{c}\text { Temperatura } \\
\left({ }^{\circ} \mathrm{C}\right)\end{array}$} & \multirow{3}{*}{$\begin{array}{c}\text { Grasa } \\
(\%)\end{array}$} & \multirow{3}{*}{$\begin{array}{c}\text { Carvacrol } \\
(\%)\end{array}$} & \multicolumn{3}{|c|}{ Valores D (min) } \\
\hline & & & & \multicolumn{2}{|c|}{ Experimental } & \multirow{2}{*}{$\frac{\text { Predicho }}{\text { LS }^{\mathrm{d}}}$} \\
\hline & & & & Promedio $^{b}$ & $\mathrm{DE}^{\mathrm{c}}$ & \\
\hline 1 & 57 & 8 & 0.4 & 5.35 & 0.66 & 4.39 \\
\hline 2 & 57 & 8 & 1.6 & 4.90 & 0.32 & 4.14 \\
\hline 3 & 57 & 17 & 0.4 & 7.71 & 0.44 & 7.64 \\
\hline 4 & 57 & 17 & 1.6 & 4.57 & 0.10 & 4.83 \\
\hline 5 & 63 & 8 & 0.4 & 0.43 & 0.03 & 0.36 \\
\hline 6 & 63 & 8 & 1.6 & 0.42 & 0.02 & 0.34 \\
\hline 7 & 63 & 17 & 0.4 & 0.63 & 0.00 & 0.63 \\
\hline 8 & 63 & 17 & 1.6 & 0.53 & 0.05 & 0.40 \\
\hline 9 & 55 & 12.5 & 1.0 & 14.45 & 0.64 & 14.22 \\
\hline 10 & 65 & 12.5 & 1.0 & 0.18 & 0.00 & 0.21 \\
\hline 11 & 60 & 5 & 1.0 & 0.88 & 0.01 & 1.14 \\
\hline 12 & 60 & 20 & 1.0 & 2.04 & 0.08 & 2.08 \\
\hline 13 & 60 & 12.5 & 0 & 1.62 & 0.00 & 1.37 \\
\hline 14 & 60 & 12.5 & 2.0 & 0.85 & 0.03 & 0.89 \\
\hline 15 & 60 & 12.5 & 1.0 & 0.88 & 0.24 & 1.10 \\
\hline 16 & 60 & 12.5 & 1.0 & 0.98 & 0.07 & 1.10 \\
\hline 17 & 60 & 12.5 & 1.0 & 0.88 & 0.13 & 1.10 \\
\hline 18 & 60 & 12.5 & 1.0 & 0.97 & 0.12 & 1.10 \\
\hline 19 & 60 & 12.5 & 1.0 & 1.06 & 0.08 & 1.10 \\
\hline 20 & 60 & 12.5 & 1.0 & 1.25 & 0.28 & 1.10 \\
\hline 21 & 55 & 5 & 0 & 13.50 & 0.10 & 13.83 \\
\hline 22 & 57 & 5 & 0 & 4.05 & 0.72 & 4.46 \\
\hline 23 & 60 & 5 & 0 & 1.16 & 0.02 & 1.07 \\
\hline 24 & 63 & 5 & 0 & 0.41 & 0.12 & 0.36 \\
\hline 25 & 65 & 5 & 0 & 0.17 & 0.01 & 0.21 \\
\hline
\end{tabular}

a Las corridas 1-8, son puntos factoriales, las 9-14 son puntos axiales, las 15-20 son puntos centrales, y las 21-25 son puntos control del DCC b Promedio de dos replicas, cada una por duplicado c Desviación estándar d Límite superior del intervalo de confianza (95\%) del valor predicho por el modelo
Tabla 3. Coeficientes de regresión estimados (CR) y análisis de varianza del modelo de regresión para E. coli 0157: H7 en carne molida de res con diferentes niveles de grasa y carvacrol, cocinadas a diferentes temperaturas Table 3. Estimated regression coefficient (RC), and analysis of variance of the regression model for $\mathrm{E}$. coli $\mathrm{O} 157: \mathrm{H} 7$ in ground beef with different fat and carvacrol levels, cooked at different temperatures

\begin{tabular}{lcccc}
\hline Factor & CR & DE & Valor F & Probabilidad \\
\cline { 2 - 5 } & & & & \\
\hline Intercepto & 0.097 & 0.058 & 179.43 & $<0.0001$ \\
\hline Temperatura & -1.25 & 0.039 & 1032.14 & $<0.0001$ \\
\hline Grasa & 0.18 & 0.046 & 14.81 & $<0.0012$ \\
\hline Carvacrol & -0.13 & 0.046 & 7.8 & 0.012 \\
Grasa x Carvacrol & -0.1 & 0.045 & 4.88 & 0.0403 \\
Temperaturax & 0.16 & 0.039 & 17.045 & 0.0006 \\
Temperatura & & & & \\
Grasax Grasa & 0.12 & 0.043 & 7.64 & 0.0128 \\
Valor P de la regresión & & & & $<0.0001$ \\
\hline Falta de ajuste & & & & 0.206 \\
\hline$R^{2}$ & & & & 0.9836 \\
\hline
\end{tabular}

$\mathrm{DE}=$ Desviación estándar

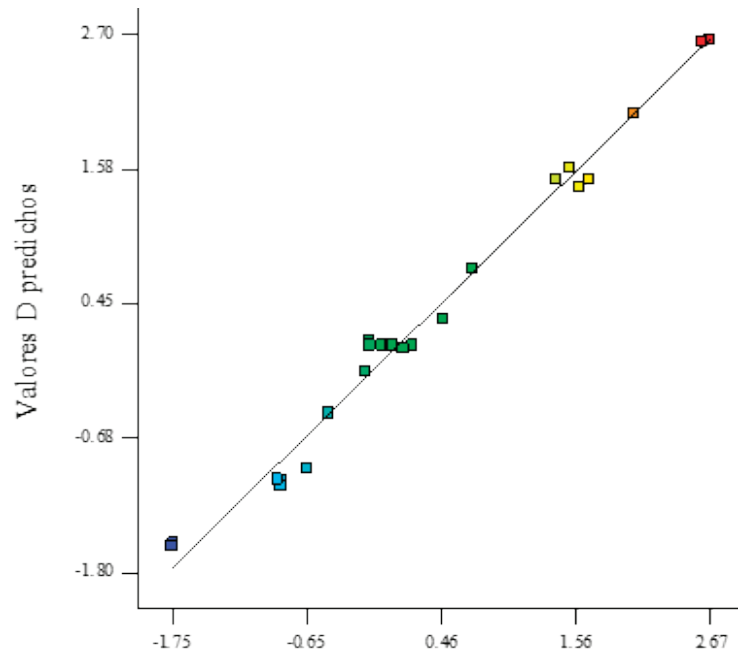

Val ores D experimental es

Figura 2. Concordancia entre los valores $D$ experimentales y predichos por el modelo para E. coli O157: H7 en carne molida de res con diferentes niveles de grasa y carvacrol, cocinadas a diferentes temperaturas.

Figure 2. Agreement between experimental and predicted $D$ values for $E$. coli O157: H7 in ground beef with different fat and carvacrol levels, cooked at different temperatures. 
De acuerdo a los coeficientes de regresión de cada uno de los términos del modelo presentados en la ecuación anterior se aprecia que la temperatura $(T=-1.25)$ es el factor más influyente en la resistencia térmica de $E$. coli 0157: $H 7$, seguido de la grasa ( $G=0.18$ ) y el carvacrol $(C=-0.13)$. También son significativos los efectos de interacción entre la grasa y el carvacrol (GC) y los efectos cuadráticos de la temperatura $\left(T^{2}\right)$ y la grasa $\left(G^{2}\right)$.

Los coeficientes de regresión negativo para la temperatura y para el carvacrol indican que a medida que éstos aumentan, el valor $\mathrm{D}$ disminuye. Por el contrario, la adición de grasa a la carne molida muestra un comportamiento opuesto (coeficiente de regresión positivo), con respecto al valor D estimado por el modelo. Estos comportamientos se pueden observar gráficamente en la Figura 3a y Figura 3b donde se muestran los efectos combinados de temperatura-grasa, y temperatura-carvacrol, respectivamente. El efecto cuadrático de la temperatura se evidencia en estas mismas figuras por la curvatura observada alrededor de $\operatorname{los} 61^{\circ} \mathrm{C}$, temperatura a partir de la cual E. coli 0157: $\mathrm{H} 7$ no ofrece resistencia al calor.

El efecto de la temperatura en la inactivación de E. coli 0157: H7 se debe principalmente a la acción del calor sobre las membranas celulares y las proteínas. La configuración de la membrana se ve afectada porque los fosfolípidos que componen la bicapa, cambian su estructura haciéndola más fluida y más permeable a los componentes del medio. También se desnaturalizan las proteínas embebidas en la membrana celular y el citosol, causando un grave daño en la integridad de la membrana y el metabolismo celular.

Smith et al. (2001), realizaron la inactivación térmica de E. coli O157: $\mathrm{H} 7$ en carne molida de res con condiciones de temperatura $\left(55,58,61\right.$ y $\left.63^{\circ} \mathrm{C}\right)$ y grasas $(4.8 \%)$ similares a los controles del presente estudio $\left(55,57.5,60\right.$ y $62.5^{\circ} \mathrm{C}$ con $5 \%$ de grasa). Los valores $\mathrm{D}$ encontrados: $20.08 \mathrm{~min}$ a $55^{\circ} \mathrm{C}, 1.22$ min a $58{ }^{\circ} \mathrm{C}, 0.32 \min$ a $61^{\circ} \mathrm{C}$ y 0.16 min a $63^{\circ} \mathrm{C}$, muestran que, al aumentar la temperatura de cocción, el tiempo necesario para lograr la muerte térmica el patógeno se reduce significativamente.

La grasa tiene un efecto protector contra la inactivación térmica de $E$. coli 0157: $\mathrm{H} 7$ en carne molida ( $G=0.18)$, por lo tanto, un aumento en ella causa la misma tendencia en el valor $D$. El efecto de la grasa sobre el valor $D$ es solo a temperaturas inferiores a $60{ }^{\circ} \mathrm{C}$, a partir de este valor no hay influencia en la resistencia del microorganismo al calor,

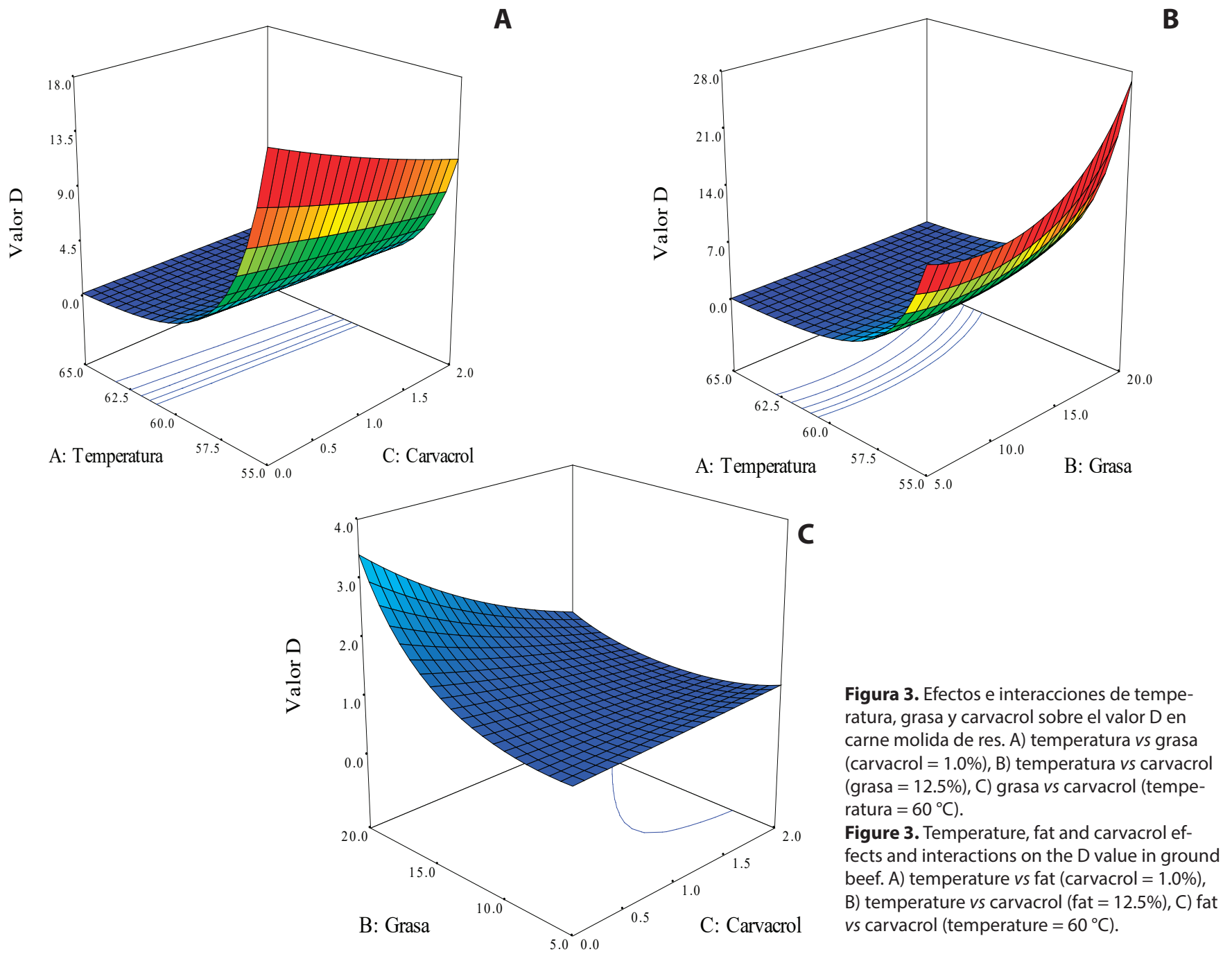


independientemente de la cantidad de grasa añadida (Figura 3a). A partir de los $60^{\circ} \mathrm{C}$, la temperatura tiene un efecto mayor sobre la mortalidad de los patógenos que no puede ser afectado por la presencia de la grasa en la matriz cárnica. La temperatura de fusión de la grasa de res está entre 40 y $48^{\circ} \mathrm{C}$, dependiendo de la zona de la que ésta provenga. A los $60^{\circ} \mathrm{C}$ la grasa se encuentra en estado líquido y su efecto protector se ve reducido respecto a la temperatura debido a que la transmisión del calor es mucho más rápida que en estado sólido y las micelas de grasa que protegían a las bacterias del calor de la cocción se han perdido (Grompone et al., 1991).

La grasa en los productos cárnicos juega un papel importante en el sabor y la textura y su influencia en la supervivencia de los microorganismos patógenos ha sido demostrada por su acción como barrera contra el calor durante los tratamientos térmicos (Juneja et al., 2001). La matriz cárnica forma micelas de grasa que actúan como barrera y pueden contener bacterias patógenas como E. coli O157: H7. La transmisión de calor se vuelve heterogénea y se crean puntos térmicos aislados que favorecen la resistencia bacteriana y disminuyen la efectividad de los tratamientos térmicos. Las bacterias suspendidas en la grasa son más difíciles de destruir que las suspendidas en un medio acuoso, debido a una reducción en la actividad de agua. Un aumento en el contenido de grasa, disminuye el contenido de humedad lo cual altera la transferencia de calor (Ahmed et al., 1995). Cuanto mayor sea la cantidad de grasa que tiene la carne molida, se debe prestar más atención a su cocción, ya que podría proteger o enmascarar microorganismos patógenos como E. coli O157: $\mathrm{H} 7$.

En relación al efecto del carvacrol sobre el valor $\mathrm{D}$, este presenta un efecto significativo $(p<0.05)$ y de acuerdo al coeficiente en el modelo $(C=-0.13)$, el signo negativo indica que cuando se añade el carvacrol a la carne, el valor $D$ de $E$. coli O157: H7 disminuye. La influencia del carvacrol depende de la cantidad de grasa en el producto como lo indica el efecto significativo $(p<0.05)$ de la interacción grasa-carvacrol $(G C=$ 0.1 ), este comportamiento se aprecia claramente en la Figura $3 c$. A bajas concentraciones de grasa, de alrededor del $5 \%$, el efecto del carvacrol no es tan marcado como a altas concentraciones. Esta tendencia se debe a las propiedades químicas del carvacrol que lo hacen más soluble en compuestos no polares, ya que tiene un coeficiente de partición $\log \mathrm{P}=3.49$ (Nazzaro et al., 2013). Un valor alto de LogP (> 2.5) indica que una sustancia es más hidrofóbica. Por lo tanto, el carvacrol es más soluble en sustancias orgánicas como la grasa que en el agua (Baluja et al., 2017).

La acción antimicrobiana del carvacrol se basa en su interacción con las membranas bacterianas, provocando daños irreparables en la estructura al elevar la permeabilidad y la despolarización (Ait-Ouazzou et al., 2013). Se ha observado que añadir carvacrol al medio de crecimiento de $E$. coli provoca un aumento del flujo de ATP y de iones $\mathrm{PO}_{4}$ y K' , del interior hacia el exterior de la membrana bacteriana (Can Baser, 2008).

\section{Efecto de la grasa y carvacrol sobre la estimación de los valores $z$}

El impacto de la adición de diferentes cantidades de grasa y carvacrol sobre la resistencia térmica de $E$. coli 0157: H7 en carne molida se muestra en la Figura 4. El valor $z$ obtenido para cada combinación de carvacrol y grasa en los rangos de 0 al $2 \%$ y de 5 al 20\%, respectivamente, permaneció constante, siendo éste de $5.47^{\circ} \mathrm{C}$. Este valor indica que para lograr una reducción de un logaritmo en el valor $D$, la temperatura de tratamiento térmico del alimento debe aumentar en $5.47^{\circ} \mathrm{C}$. El valor $z$ es un factor que se calcula para cada alimento y cada microorganismo a partir del valor $D, y$ se utiliza para la implementación de tratamientos térmicos y el cálculo de la letalidad sobre los microorganismos de interés en el alimento.

\section{Optimización y validación de la condición óptima predi- cha por el modelo de regresión}

La optimización de las condiciones de proceso para carne molida res se realizó de acuerdo a lo descrito en la sección de materiales y métodos. Las condiciones óptimas de proceso que minimizaron los valores $D$ fueron: tratamiento térmico de $63^{\circ} \mathrm{C}$ de carne molida de res adicionada con $11 \%$ de grasa y $1.6 \%$ de carvacrol. El valor $\mathrm{D}$ predicho por el modelo para estas condiciones fue de $0.30 \mathrm{~min}$. Para determinar cuan preciso es el modelo de regresión en la predicción de los valores de $D$ en carne molida de res, se comparó el valor D experimental ( $n=8$, rango de 0.20 a $0.36 \mathrm{~min}$ ) contra el valor $D$ predicho por el modelo $(0.30 \mathrm{~min})$ en las condiciones óptimas y no se encontraron diferencias $(p>0.05)$. A partir de este resultado se puede afirmar que el modelo obtenido es capaz de predecir con una probabilidad mayor al $95 \%$ el valor D para las condiciones evaluadas. Validar el modelo

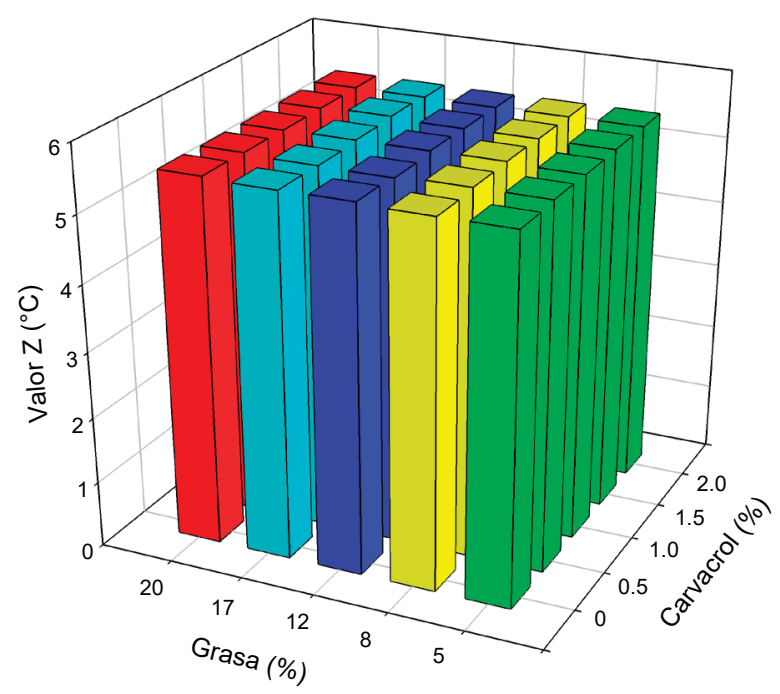

Figura 4. Valores $z$ de $E$. coli $0157: \mathrm{H} 7$ calculados a partir de los valores D estimados en carne molida de res adicionada con carvacrol ( 0 al $2 \%$ ) y grasa (5 al 20\%).

Figure 4. E. coli 0157: $\mathrm{H} 7 \mathrm{z}$-values calculated from estimated D-values in ground beef added with 0 to $2 \%$ carvacrol and 5 to $20 \%$ fat. 
permite demostrar la eficacia del mismo para ser usado en procesos térmicos con un elevado grado de confianza.

\section{CONCLUSIONES}

En el presente trabajo se logró obtener un modelo de regresión por MSR que describe el comportamiento de la resistencia térmica de $E$. coli 0157: $\mathrm{H} 7$ en función de la temperatura $\left(55-65^{\circ} \mathrm{C}\right)$, y la concentración de grasa (5-20\%) y carvacrol (0-2\%) en carne molida de res. La temperatura fue el factor que más influyó en la resistencia térmica del patógeno, seguido de la grasa y el carvacrol. Tanto la temperatura como el carvacrol disminuyeron la resistencia térmica del $E$. coli $0157: \mathrm{H7}$, mientras que la grasa tuvo un efecto protector. Este efecto protector fue revertido por la adición de carvacrol, tal como lo indica el efecto de interacción entre estos factores. Las condiciones óptimas que minimizan el valor $D$ fueron: $63{ }^{\circ} \mathrm{C}, 11 \%$ de grasa y $1.6 \%$ de carvacrol. Con estas condiciones, el valor $\mathrm{D}$ predicho por el modelo fue de 0.30 min. Durante la validación del modelo, se demostró que no existen diferencias entre el valor predicho por el modelo y los obtenidos experimentalmente. El procesador de alimentos puede seleccionar cualquier combinación de temperatura, grasa y carvacrol, en los límites establecidos, para estimar la reducción logarítmica de E. coli O157: H7 en carne molida de res.

\section{AGRADECIMIENTOS}

Los autores agradecen al Consejo Nacional de Ciencia y Tecnología por la beca otorgada al primer autor durante sus estudios de Maestría en Ciencias.

\section{REFERENCIAS}

Ahmed, N. M., Conner, D. E., Huffman, D. L. 1995. Heat-resistance of Escherichia coli 0157: H7 in meat and poultry as affected by product composition. Journal of Food Science. 60: 606610.

Ait-Ouazzou, A., Espina, L., Gelaw, T., de Lamo-Castellví, S., Pagán, R., García-Gonzalo, D. 2013. New insights in mechanisms of bacterial inactivation by carvacrol. Journal of Applied Microbiology. 114: 173-185.

Baluja, S., Kulshrestha, A., Movalia, J. 2017. 1-Octanol-water partition coefficient of some cyanopyridine and chalcone compounds. Revista Colombiana de Ciencias QuímicoFarmacéuticas. 46: 342-356.

Baranyi, J., Roberts, T. A. 1995. Mathematics of predictive food microbiology. International Journal of Food Microbiology. 26: 199-218.

Can Baser, K. 2008. Biological and pharmacological activities of carvacrol and carvacrol bearing essential oils. Current Pharmaceutical Design. 14: 3106-3119.

Cashman, K. D., Hayes, A. 2017. Red meat's role in addressing 'nutrients of public health concern'. Meat Science. 132: 196203.

Centers for Disease Control and Prevention. 2020. E. coli (Escherichia coli). Disponible en: https://www.cdc.gov/ecoli/ index.html

Grompone, M. A. 1991. Propiedades físicas y químicas de las grasas bovinas fraccionadas e interesterificadas. Grasas y Aceites, 42: 349-355.
Juneja, V. K., Eblen, B., Marks, H. 2001. Modeling non-linear survival curves to calculate thermal inactivation of Salmonella in poultry of different fat levels. International Journal of Food Microbiology. 70: 37-51.

Juneja, V. K., Friedman, M. 2008. Carvacrol and cinnamaldehyde facilitate thermal destruction of Escherichia coli 0157: $\mathrm{H} 7$ in raw ground beef. Journal of Food Protection. 71: 1604-1611.

Juneja, V. K., Novak, J. S. 2003. Heat resistance of Escherichia coli O157: H7 in cook-in-bag ground beef as affected by $\mathrm{pH}$ and acidulant. International Journal of Food Science \& Technology. 38: 297-304.

Juneja, V. K., Thippareddi, H., Friedman, M. 2006. Control of Clostridium perfringens in cooked ground beef by carvacrol, cinnamaldehyde, thymol, or oregano oil during chilling. Journal of Food Protection. 69: 1546-1551.

Juneja, V. K., Yadav, A. S., Hwang, C.-A., Sheen, S., Mukhopadhyay, S., Friedman, M. 2012. Kinetics of thermal destruction of Salmonella in ground chicken containing transcinnamaldehyde and carvacrol. Journal of Food Protection. 75: 289-296.

Kim, J.-S., Lee, M.-S., Kim, J. H. 2020. Recent Updates on Outbreaks of Shiga Toxin-Producing Escherichia coli and Its Potential Reservoirs. Frontiers in Cellular and Infection Microbiology. 10: 1-10.

Lin, C.-T. J. 2018. Self-reported methods used to judge when a hamburger is ready at-home in a sample of US adults. Food Control. 91: 181-184.

Lyon, B., Berry, B., Soderberg, D., Clinch, N. 2000. Visual color and doneness indicators and the incidence of premature brown color in beef patties cooked to four end point temperatures. Journal of Food Protection. 63: 1389-1398.

Mongomery, C. D. 2013. Design and analysis of experiments (eighth ed.): John Wiley \& Sons Inc.

Nazzaro, F., Fratianni, F., De Martino, L., Coppola, R., De Feo, V. 2013. Effect of essential oils on pathogenic bacteria. Pharmaceuticals. 6: 1451-1474.

Ríos-Flores, J. A. y Castillo-Arce, M. L. 2015. La competitividad de la carne fresca de res mexicana en el mercado estadounidense. Estudios Fronterizos, 16: 221- 245.

Scandorieiro, S., de Camargo, L. C., Lancheros, C. A., YamadaOgatta, S. F., Nakamura, C. V., de Oliveira, A. G., Andrade, C. G., Duran, N., Nakazato, G., Kobayashi, R. K. 2016. Synergistic and additive effect of oregano essential oil and biological silver nanoparticles against multidrug-resistant bacterial strains. Frontiers in Microbiology. 7: 1-14.

Smith, S., Maurer, J., Orta-Ramirez, A., Ryser, E., Smith, D. 2001. Thermal inactivation of Salmonella spp., Salmonella typhimurium DT104, and Escherichia coli 0157: H7 in ground beef. Journal of Food Science. 66: 1164-1168.

Surendran Nair, M., Lau, P., Belskie, K., Fancher, S., Chen, C.-H., Karumathil, D. P., Yin, H.-B., Liu, Y., Ma, F., Upadhyaya, I. 2016. Potentiating the heat inactivation of Escherichia coli 0157: H7 in ground beef patties by natural antimicrobials. Frontiers in Microbiology. 7: 1-8.

USDA-FSIS. (2020). U.S. Department of Agriculture, Food Safety Inspection Service. Disponible en: https://www.fsis.usda. gov/wps/portal/fsis/topics/food-safety-education/getanswers/foodsafety-fact-sheets/meat-preparation/color-ofcooked-ground-beef-as-itrelates-to-doneness 